\title{
Ventrolateral Prefrontal Cortex Activation and Attentional Bias in Response to Angry Faces in Adolescents With Generalized Anxiety Disorder
}

Christopher S. Monk, Ph.D.

Eric E. Nelson, Ph.D.

Erin B. McClure, Ph.D.

Karin Mogg, Ph.D.

Brendan P. Bradley, Ph.D.

Ellen Leibenluft, M.D.

R. James R. Blair, Ph.D.

Gang Chen, Ph.D.

Dennis S. Charney, M.D.

Monique Ernst, M.D., Ph.D.

Daniel S. Pine, M.D.

\begin{abstract}
Objective: While adolescent anxiety disorders represent prevalent, debilitating conditions, few studies have explored their brain physiology. Using event-related functional magnetic resonance imaging (fMRI) and a behavioral measure of attention to angry faces, the authors evaluated differences in response between healthy adolescents and adolescents with generalized anxiety disorder.
\end{abstract}

Method: In the primary trials of interest,
18 adolescents with generalized anxiety
disorder and 15 comparison subjects of
equivalent age/gender/IQ viewed angry/
neutral face pairs during fMRI acquisition.
Following the presentation of each face
pair, subjects pressed a button to indicate
whether a subsequent asterisk appeared
on the same (congruent) or opposite (in-
congruent) side as the angry face. Reac-
tion time differences between congruent
and incongruent face trials provided a
measure of attention bias to angry faces.
Results: Relative to the comparison subjects, patients with generalized anxiety disorder manifested greater right ventrolateral prefrontal cortex activation to trials containing angry faces. Patients with generalized anxiety disorder also showed greater attention bias away from angry faces. Ventrolateral prefrontal cortex activation differences remained evident when differences in attention bias were covaried. Finally, in an examination among patients of the association between degree of anxiety and brain activation, the authors found that as ventrolateral prefrontal cortex activation increased, severity of anxiety symptoms diminished.

Conclusions: Adolescents with generalized anxiety disorder show greater right ventrolateral prefrontal cortex activation and attentional bias away from angry faces than healthy adolescents. Among patients, increased ventrolateral prefrontal cortex activation is associated with less severe anxiety, suggesting that this activation may serve as a compensatory response.

\begin{abstract}
A dolescent generalized anxiety disorder predicts high risk for adult generalized anxiety disorder, social phobia, and major depressive disorder (1). While only one study (2) has explored neurophysiological correlates of generalized anxiety disorder in youth, considerable data in both the basic sciences and adult anxiety disorder literature form the basis for hypotheses about the roles of specific neural structures in adolescent generalized anxiety disorder (3-12).

Basic research implicates a neural circuit that includes the amygdala and ventral prefrontal cortex in threat monitoring and response $(5,9,10)$. The ventral prefrontal cortex (in particular the ventrolateral prefrontal cortex) modulates amygdala engagement to facilitate flexible attention and behavior when responding to environmental threats $(3-6,9)$. Anxiety disorders may relate to perturbations in this ventral prefrontal cortex-amygdala circuit. The only available study of anxious youth selectively imaged the amygdala in 10 patients and 10 comparison subjects and reported that patients showed greater response to threat-related facial expressions (2). Among adults, patients with anxiety disorders
\end{abstract}

have shown increased amygdala activation and abnormal ventral prefrontal cortex responses $(8,12-15)$. These neural abnormalities may disrupt the regulatory processes of vigilance and attention to threat-related stimuli.

Increased vigilance and perturbed attention are prominent features of pediatric and adult anxiety disorders (1623). Thus, brain-based differences between anxious patients and comparison subjects may reflect anxiety-related differences in attentional processes. For example, in contrast with healthy subjects, anxious patients may shift attention away from threatening stimuli, which could either reflect or affect between-group differences in brain activation. Therefore, when examining between-group differences in neural responses, it may be advantageous to monitor attention and examine effects of attention on between-group differences in activation.

The visual task used in this study provides an index of threat-related perturbations in attention (16, 17, 20-22). In previous studies that used this task, adult patients with generalized anxiety disorder and other anxiety disorders 
TABLE 1. Demographic and Clinical Characteristics of Adolescents With Generalized Anxiety Disorder and a Comparison Group of Healthy Adolescents

\begin{tabular}{|c|c|c|c|c|}
\hline Variable & $\begin{array}{l}\text { Healthy Group } \\
\quad(\mathrm{N}=15)\end{array}$ & & $\begin{array}{l}\text { Patient Group } \\
\quad(\mathrm{N}=18)\end{array}$ & \\
\hline & $\mathrm{N}$ & $\%$ & $\mathrm{~N}$ & $\%$ \\
\hline \multicolumn{5}{|l|}{ Gender } \\
\hline Male & 7 & 46.7 & 10 & 55.6 \\
\hline Female & 8 & 53.3 & 8 & 44.4 \\
\hline \multicolumn{5}{|l|}{ Comorbid diagnoses } \\
\hline Major depressive disorder & 0 & 0.0 & 9 & 50.0 \\
\hline Social phobia & 0 & 0.0 & 10 & 55.6 \\
\hline \multirow[t]{2}{*}{ Separation anxiety disorder } & 0 & 0.0 & 8 & 44.4 \\
\hline & Mean & SD & Mean & SD \\
\hline Age & 13.53 & 2.41 & 12.28 & 2.05 \\
\hline IQ & 115.13 & 9.77 & 109.00 & 12.65 \\
\hline
\end{tabular}

orient attention toward angry faces (i.e., the "threat") more strongly than healthy adults (21-23). It is interesting that in a study of children with posttraumatic stress disorder (PTSD), the visual task findings were opposite to those in adults: pediatric patients displayed a greater attentional bias away from threat, in comparison with healthy children (16). While preliminary, these findings suggest that anxiety disorders may involve systematic disruptions in threat biases that vary across development.

For the present functional magnetic resonance imaging (fMRI) investigation, we used the visual task to examine differences between adolescents with generalized anxiety disorder and healthy adolescents. Consistent with prior studies, we hypothesized that adolescents with generalized anxiety disorder would show abnormal ventral prefrontal cortex activation and increased amygdala activation relative to healthy comparison subjects in response to angry faces. We also hypothesized that adolescents with generalized anxiety disorder would exhibit abnormal attentional bias to angry faces.

\section{Method}

\section{Participants}

Thirty-three adolescents participated in this study. Health and cognitive status of all participants was determined with a physical examination and an IQ test. The Schedule for Affective Disorders and Schizophrenia (K-SADS) was administered by trained clinicians. Based on an independent study, each clinician had been shown to have excellent reliability (kappa $>0.75$ ) with senior clinicians for all diagnoses. Demographic characteristics for all study subjects are provided in Table 1. The NIMH Institutional Review Board approved all procedures, and all adolescents/parents provided written assent/consent.

The primary inclusion criterion was a diagnosis of generalized anxiety disorder in an adolescent (age 9-17 years); the generalized anxiety disorder diagnosis had to be the explicit focus of treatment. Other inclusion criteria were similar to those in published studies of pediatric anxiety disorders (24-26). Specifically, these included 1) clinically significant symptoms of anxiety (Pediatric Anxiety Rating Scale [27] score $\geq 10$ ); 2) Children's Global Assessment Scale score $<60 ; 3)$ desire for outpatient treatment; and 4) persistent anxiety (consistent Pediatric Anxiety Rating Scale score over a 3-week period during which supportive psychoeducational therapy was provided).

Exclusion criteria were 1) current use of any psychoactive substance (for patients, usage of any psychoactive substance since the onset of the anxiety disorder); 2) current Tourette's syndrome, obsessive-compulsive disorder, PTSD, conduct disorder, exposure to extreme trauma, or suicidal ideation; 3 ) lifetime history of mania, psychosis, or pervasive developmental disorder; or 4) $\mathrm{IQ}<70$. Major depressive disorder was not considered exclusionary, since longitudinal and family-based studies have noted strong relationships between adolescent generalized anxiety disorder and major depressive disorder. Secondary fMRI analyses compared activation between groups with and without major depressive disorder as well as social phobia to determine the degree to which comorbidity moderated findings in patients with generalized anxiety disorder.

\section{Visual Task}

We used the same procedures and stimuli as previously described (28) (Figure 1). In brief, the trials began with a $500-\mathrm{msec}$ central fixation point. Two faces then appeared for $500 \mathrm{msec}$. These were replaced by an asterisk in one hemi-field for 1100 msec. Participants were instructed to press one button with their thumb if the asterisk appeared on the left or a second button with their index finger if the asterisk appeared on the right. The intertrial interval was $1900 \mathrm{msec}$. Before scanning, participants were trained on the task.

Participants saw 80 actors, each presented twice, for a total of 160 trials (Figure 1). An additional 40 blank trials (no faces and no asterisks) were presented to serve as the primary comparison for documenting between-group differences in activation to the angry faces.

For the behavioral measure of attention, there were two conditions of interest: 1) congruent trials, in which an angry/neutral face pair was followed by an asterisk on the same side on which the angry face had appeared; and 2) incongruent trials, in which an angry/neutral face pair was followed by an asterisk on the same side on which the neutral face had appeared. Three other control conditions were included: happy/neutral face pair congruent trials, happy/neutral face pair incongruent trials, and neutral/neutral face pairs. There were 32 trials for each of the five conditions. Trial presentation order was randomly determined for each subject. Equal numbers of replicates displayed the emotional face on right and left hemi-fields.

The task was programmed by using E-Prime versions 1.0 and 1.1 by Psychological Software Tools (Pittsburgh, Pa.) and was displayed on Avotec Silent Vision Glasses (Stuart, Fla.). The button response system was developed by MRI Devices (Waukesha, Wisc.). 
FIGURE 1. Visual Tasks Presented During fMRI Acquisition to Adolescents With Generalized Anxiety Disorder and a Comparison Group of Healthy Adolescents ${ }^{a}$

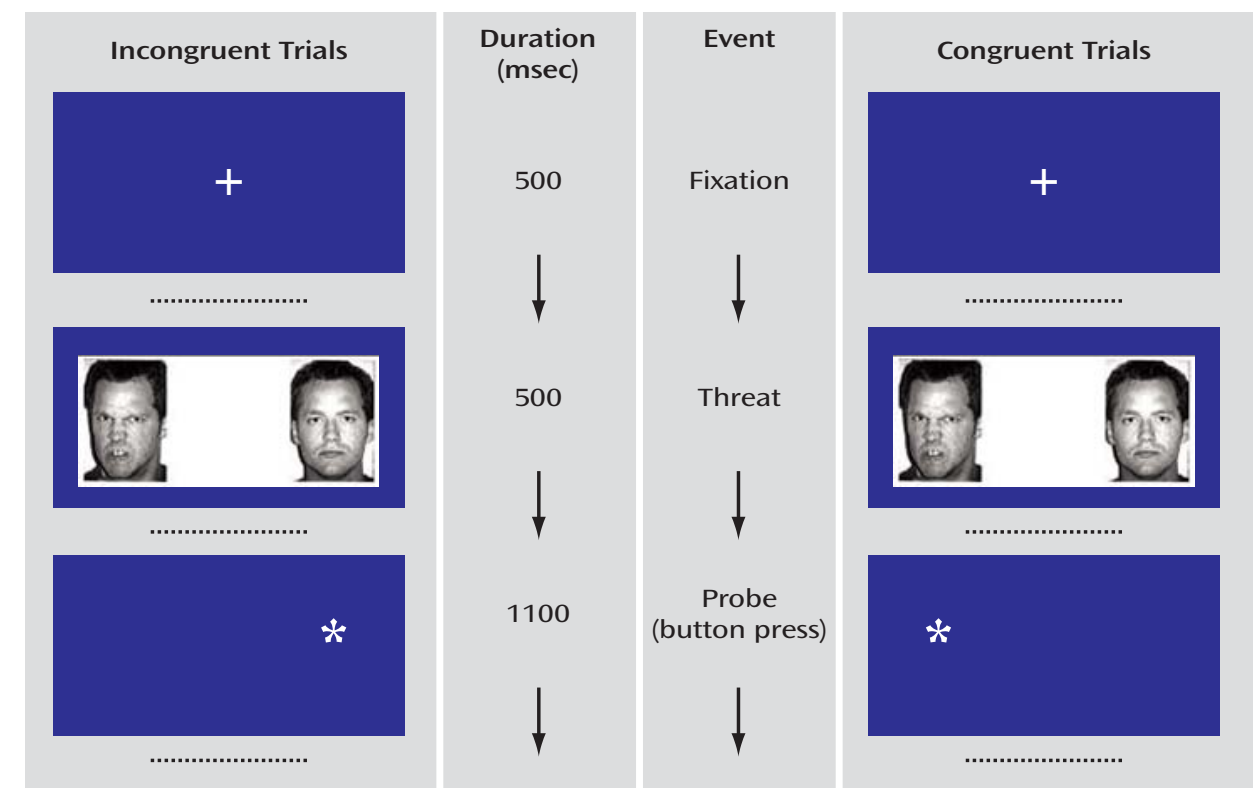

a The only difference between the two trials is the location of the probe relative to the threat. For congruent trials, the asterisk (the "probe") following the presentation of the angry/neutral face pair was on the same side on which the angry face (the "threat") had appeared. For incongruent trials, the asterisk was on the same side on which the neutral face had appeared. The same actor always modeled the two expressions in the face pair. Trials with happy/neutral and neutral/neutral face pairs were also conducted.

\section{Functional MRI Procedures and Analyses}

Imaging took place over approximately a 1-year period. We used a GE 3T scanner to acquire images with 29 contiguous 3.3$\mathrm{mm}$ axial slices parallel to the anterior commissure/posterior commissure line. We used echo-planar, single-shot gradient echo $\mathrm{T} 2 *$ weighting $(\mathrm{TR}=2300 \mathrm{msec}$; $\mathrm{TE}=23 \mathrm{msec}$; field of view $=240$ $\mathrm{mm}$; $64 \times 64$ matrix; $3.3 \times 3.75 \times 3.75 \mathrm{~mm}$ voxel). High-resolution T1weighted volumetric scans used a magnetization-prepared gradient echo sequence (MP-RAGE): 180 1.0-mm axial slices; field of view=256 mm; number of excitations $=1$; $T R=11.4 \mathrm{msec}$; $\mathrm{TE}=4.4$ msec; matrix $=256 \times 256$; TI=300 msec; bandwidth $130 \mathrm{~Hz} /$ pixel=33 $\mathrm{kHz}$ for 256 pixels; in-plane resolution $=1 \mathrm{~mm}^{3}$.

Functional imaging data were analyzed using AFNI software version 2.56b (available at http://afni.nimh.nih.gov/afni) (29). Participants were excluded if there was movement greater than 2.5 $\mathrm{mm}$ in any direction. Movement was mitigated by registering the images to one volume in each run. Subject data were smoothed with a $6-\mathrm{mm}$ full width at half maximum isotropic Gaussian filter. Incorrect trials and trials in which the reaction time for the button response was $<200 \mathrm{msec}$ or $>800 \mathrm{msec}$ were removed from the fMRI analysis.

A random effects fMRI data analysis was conducted by using a two-level procedure. At the first (subject) level, data from each subject were separately submitted to multiple regression analyses using the 3dDeconvolve module in AFNI. For the five conditions (angry/neutral congruent, angry/neutral incongruent, happy/ neutral congruent, happy/neutral incongruent, and neutral/neutral), five vectors were created containing the onset time of each trial for each condition (blank trials were modeled as an implicit baseline). Time points for trials in which the subject responded incorrectly or did not respond were placed in a separate vector included in the multiple regression model as a nuisance covariate. Using a gamma variate (30), vectors of stimulus timing were transformed into reference waveforms of response function for multiple regression, and coefficients were produced for each con- dition from each subject. Comparison of coefficients for given conditions yielded contrast values. The primary effect of interest for the fMRI analysis was response to angry faces. Therefore, contrasts were established to examine activation to angry faces relative to baseline. Before individual subject data sets were subjected to a group level analysis, individual anatomical data sets were converted to Talairach space. The underlying transformation was then applied to that same individual's functional data in order to convert those images as well. The second (group) level analysis involved conducting a regression analysis using the AFNI module 3DregAna on the main contrast of interest: angry versus baseline trials to evaluate the neurophysiological response with age as a covariate following previous work (16). For comparison, we used the same procedures to examine responses to happy faces versus baseline and neutral faces versus baseline.

We employed two thresholds to evaluate the fMRI results. For the first threshold, we used the standard whole-brain $\mathrm{p}<0.001$ twotailed t test uncorrected for multiple comparisons throughout the brain (31). As a second step if activation in an area was significant at $\mathrm{p}<0.001$, we also used a Monte Carlo simulation (32) to separately control for multiple comparisons within the primary areas of interest, the ventral frontal area (anterior to anterior commissure and ventral to genu of corpus callosum) and the amygdala. In the Monte Carlo simulation, the threshold for considering a given voxel significant was $\mathrm{p}<0.01$. All voxels within the ventral frontal area that reached this level of significance were included in the Monte Carlo simulation. For the overall Monte Carlo simulation, we used the $\mathrm{p}<0.05$ level for statistical significance.

\section{Behavioral Data Analysis}

The same deviant trials were removed as in the fMRI analysis. Participants were excluded from data analysis if fewer than $75 \%$ of the responses were correct or within the accepted reaction time range.

Attentional bias scores reflected the difference between mean reaction times for incongruent and congruent trials, such that 
FIGURE 2. Ventrolateral Prefrontal Cortex Activation in Healthy Adolescents and Adolescents With Generalized Anxiety Disorder by Depressive Comorbidity and Anxiety Symptom Severity ${ }^{\mathrm{a}}$

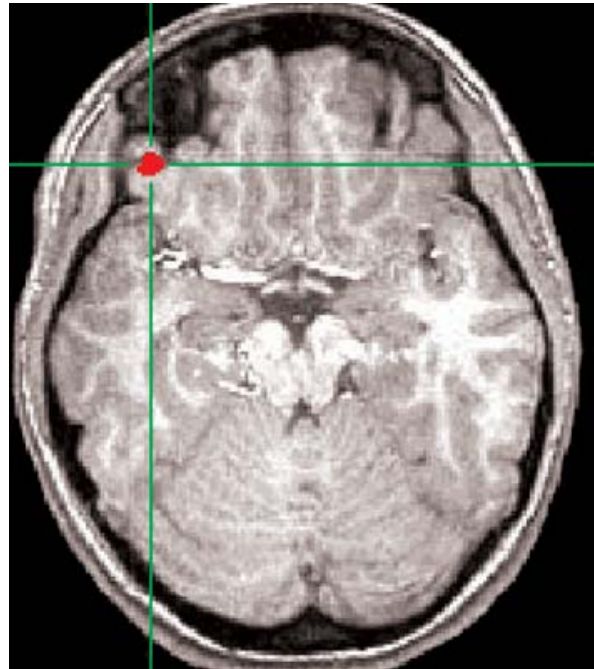

Site in Right Ventrolateral Prefrontal Cortex Where Activation Was Greater in Adolescents With Generalized Anxiety Disorder Than in Comparison Subjects ${ }^{a}$

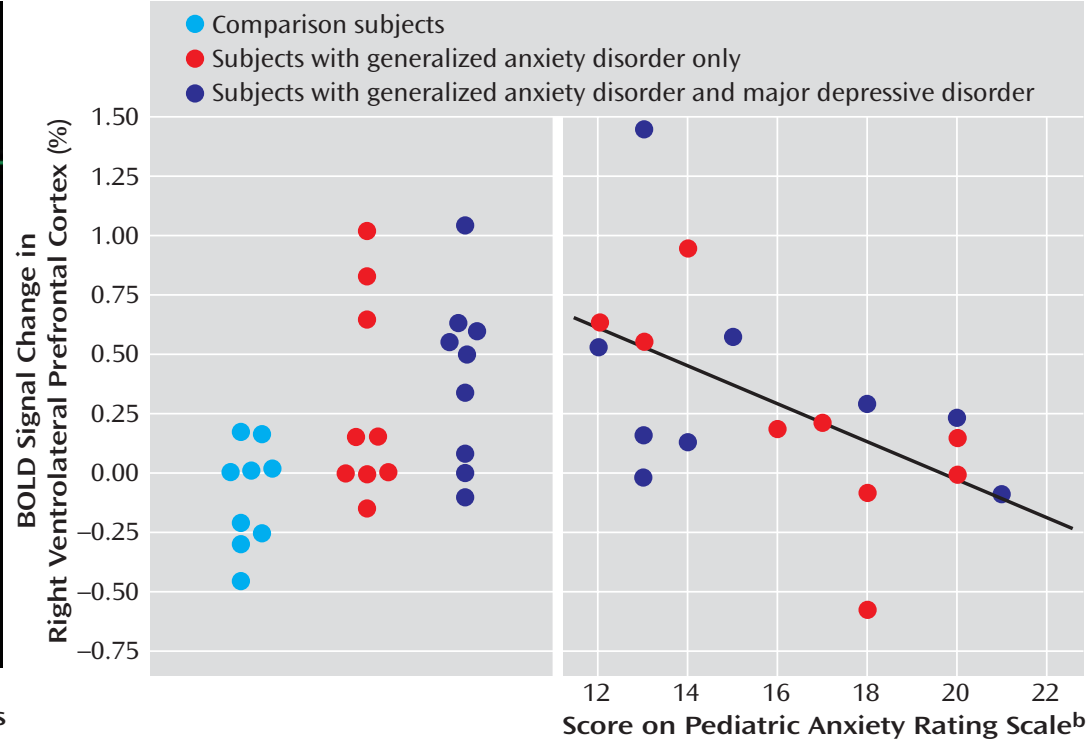

${ }^{a}$ Coordinates $(x, y, z)$ are 40,34 , and -12 .

${ }^{b}$ Blood oxygen level-dependent signal response in the right ventrolateral prefrontal cortex of the generalized anxiety disorder patients was inversely correlated with severity of anxiety symptoms $(r=-0.55, \mathrm{df}=16, \mathrm{p}<0.05)$.

positive values indicate bias toward angry faces and negative values indicate bias away from angry faces $(16,22)$. A comparable analysis examined bias for happy faces. An analysis of variance with age entered as a covariate was used to compare group differences in attentional bias, following previous work (16) and to be consistent with the fMRI analysis.

\section{Results}

The primary fMRI and behavioral analyses examined whether there were fMRI activation and reaction time differences between the generalized anxiety disorder and comparison groups in response to angry faces. In addition, we compared activation between groups for responses to happy and neutral faces. Secondary analyses evaluated the degree to which differences in the primary analysis were influenced by either task performance or comorbidity.

\section{Activation Results}

Differential activation between the groups was examined for the contrast of angry faces versus baseline. In this analysis, generalized anxiety disorder adolescents showed greater activation in the right ventrolateral prefrontal cortex $(\mathrm{t}=3.91, \mathrm{df}=30, \mathrm{p}<0.001)$ (Figure 2). The Monte Carlo simulation was significant at $\mathrm{p}<0.05$ corrected for multiple comparisons of the region of interest. No effects in the amygdala were found. No significant differences in activation were found between the two groups in response to either happy or neutral faces relative to baseline.

\section{Behavioral Results}

Adolescents with generalized anxiety disorder, relative to comparison subjects, showed a greater attentional bias away from angry faces $(\mathrm{F}=4.90, \mathrm{df}=1,30, \mathrm{p}<0.05)$. For patients, attentional bias was $-6.93 \mathrm{msec}(\mathrm{SD}=32.3)$, and for the healthy subjects the bias was $10.41 \mathrm{msec}(\mathrm{SD}=21.0)$. Mean reaction times for patients were $562.4 \mathrm{msec}(\mathrm{SD}=$ $55.0)$ to angry/neutral congruent trials and $555.5 \mathrm{msec}$ $(\mathrm{SD}=53.2)$ to angry/neutral incongruent trials. Mean reaction times for healthy subjects were $523.8 \mathrm{msec}(\mathrm{SD}=89.9)$ to angry/neutral congruent trials and $534.1 \mathrm{msec}(\mathrm{SD}=$ 91.4) to angry/neutral incongruent trials. No betweengroup differences were found in reaction time overall to trials containing angry faces. Furthermore, there were no between-group differences in attention bias to happy faces, and there were no between-group differences in reaction time overall to trials with happy faces.

\section{Secondary Activation Results}

Given that there were behavioral differences in attentional bias between groups, a secondary fMRI analysis included the attentional bias measure as a covariate. In this analysis, the generalized anxiety disorder group continued to show greater activation in the previously identified right ventrolateral prefrontal cortex region $(\mathrm{t}=3.78$, $\mathrm{df}=29, \mathrm{p}<0.001$ ).

Nine generalized anxiety disorder patients had major depressive disorder, and nine had no history of major depressive disorder. We examined differences in ventrolat- 
eral prefrontal cortex activation from healthy comparison subjects separately in these two groups and relative to one another. In order to maximize the likelihood that differences would be detected between the generalized anxiety disorder and generalized anxiety disorder/major depressive disorder groups, we used uncorrected t tests and the values from the ventrolateral prefrontal cortex cluster analysis presented in Figure 2. Relative to the healthy comparison subjects, greater ventrolateral prefrontal cortex activation was seen in the generalized anxiety disorder adolescents either with major depressive disorder $(t=4.22$, $\mathrm{df}=22, \mathrm{p}<0.001)$ or without major depressive disorder $(\mathrm{t}=$ $2.89, \mathrm{df}=22, \mathrm{p}<0.01$ ). No differences in ventrolateral prefrontal cortex activation was found between the two patient groups $(\mathrm{t}=0.59, \mathrm{df}=16, \mathrm{p}=0.57)$.

Furthermore, 10 of the 18 generalized anxiety disorder patients had social phobia. To evaluate the effect of social phobia on ventrolateral prefrontal cortex activation, we employed the same liberal cluster analysis as described with major depressive disorder to maximize the likelihood of finding differences. Relative to the healthy comparison subjects, greater ventrolateral prefrontal cortex activation was seen in the generalized anxiety disorder adolescents either with social phobia $(\mathrm{t}=3.22, \mathrm{df}=21, \mathrm{p}<0.01)$ or without social phobia $(\mathrm{t}=3.70, \mathrm{df}=23, \mathrm{p}<0.01)$. No differences in ventrolateral prefrontal cortex activation were found between the two patient groups $(\mathrm{t}=0.35, \mathrm{df}=16, \mathrm{p}=0.73)$.

Finally, to examine the relationship between severity of anxiety symptoms and brain activation, we entered the patients' Pediatric Anxiety Rating Scale scores in a covariate analysis. Results revealed a significant activation in the right ventrolateral prefrontal cortex $(\mathrm{t}=3.98, \mathrm{df}=16, \mathrm{p}=$ 0.001) (Figure 2). As ventrolateral prefrontal cortex activation increased across patients, severity of anxiety symptoms diminished.

\section{Discussion}

In response to angry faces, adolescents with generalized anxiety disorder, relative to healthy adolescents, exhibited greater activation in the right ventrolateral prefrontal cortex. Furthermore, adolescents with generalized anxiety disorder showed an attentional bias away from angry faces relative to healthy adolescents. When the difference in attention was treated as a covariate in the fMRI analysis, the generalized anxiety disorder patients continued to show greater ventrolateral prefrontal cortex activation relative to comparison subjects, suggesting that differences in attention did not fully account for differences in ventrolateral prefrontal cortex engagement. Contrary to our hypothesis, patients with generalized anxiety disorder did not evidence greater amygdala activation than healthy subjects in response to angry faces. Finally, there were no differences in response to happy or neutral faces.

Abnormal activation in the ventrolateral prefrontal cortex of adolescents with generalized anxiety disorder in the current study is consistent with previous neuroimaging work on adults with various anxiety disorders, including social phobia, PTSD, and panic disorder (7, 12, 13, 33), although the direction of the effects in previous studies is inconsistent. In the present study, the enhanced generalized anxiety disorder ventrolateral prefrontal cortex activation suggests one of two possibilities: a disturbance in functioning that may be a direct neural correlate of increased anxiety, or a compensatory response designed to regulate abnormal function in another region.

Support for the first possibility comes from a study of pediatric patients with traumatic brain injury (34). This work revealed that greater damage to the ventral prefrontal cortex was associated with reduced anxiety, suggesting that this region is involved in the manifestation of anxiety symptoms. In the present study, angry faces may have induced a modicum of anxiety among patients, manifested as increased ventrolateral prefrontal cortex activation.

Concerning the second possibility, the ventral prefrontal cortex is involved in various regulatory processes $(9,35$, 36). In particular, animal work has demonstrated extensive connections between the ventral prefrontal cortex and subcortical structures, including the striatum, amygdala, and hippocampus, which are implicated in processing emotion-based information (37-39). Moreover, these connections facilitate ventral prefrontal cortex regulation of subcortical structures $(36,37)$. Similarly, imaging studies have suggested that the ventrolateral prefrontal cortex regulates activation in subcortical structures in adult humans as well $(9,40,41)$. Thus, it is possible that the ventrolateral prefrontal cortex in the patients modulates abnormal activation in another region. Differential between-group activation in the other region was not detected, possibly because the ventrolateral prefrontal cortex is effectively modulating the abnormal response. The finding that increased ventrolateral prefrontal cortex activation is associated with fewer anxiety symptoms is consistent with the possibility that the activation is compensatory. Enhanced ventrolateral prefrontal cortex activation may help generalized anxiety disorder patients more effectively regulate initial responses to anxiety-provoking stimuli, thereby reducing severity of symptoms.

The observation of greater attentional bias away from threat in adolescent anxiety disorders replicates previous work in children with PTSD (16). Both the current and the prior study document findings opposite from those found in adults. Specifically, in adults studied with the same task, anxiety disorder patients exhibit a bias toward threat faces relative to neutral faces, compared with healthy adults (21, 22). Thus, the relationship between anxiety and threat bias assessed with this task varies across development. An important direction for future work will be to uncover the mechanisms that explain the developmental variation. Monitoring eye gaze, varying intensity of threat, and manipulating presentation duration may illuminate the variables that contribute to the developmental differences. 
The current findings illustrate the importance of behavioral measures and event-related analyses in neuroimaging investigations of between-group differences. We restricted our analyses to events in which participants performed the task properly. Thus, only trials in which participants were engaged in the task were included in the analyses. Furthermore, because this task provides a measure of attention to angry faces, it was possible to evaluate whether the fMRI results were influenced by group differences in attention allocation to the angry faces. When the attentional bias measure was included in the fMRI analysis as a covariate, the between-group difference in ventrolateral prefrontal cortex activation remained significant, indicating that attention differences do not fully account for these findings.

A potential limitation of this study is that the clinical group included generalized anxiety disorder patients with comorbid major depressive disorder or social phobia. To examine whether effects were differentially influenced by these other conditions, we examined the ventrolateral prefrontal cortex activation within each of the clinical groups. Whereas both clinical subgroups showed significantly increased ventrolateral prefrontal cortex activation relative to healthy subjects in response to angry faces, the ventrolateral prefrontal cortex activation did not differ between clinical subgroups. These analyses suggest that the ventrolateral prefrontal cortex activation was not disproportionately influenced by major depressive disorder or social phobia.

A second limitation of the study is the broad age range in our relatively small group, which precluded performance of an adequately powered analysis of effects across development within adolescents. Replication in larger groups of younger and older adolescents will help to clarify the developmental progression of these findings and how they relate to anxiety.

Presented in part at the 43rd annual meeting of the American College of Neuropsychopharmacology, San Juan, Puerto Rico, Dec. 12 16, 2004. Received April 27, 2005; revisions received June 28 and Aug. 8, 2005; accepted Sept. 19, 2005. From the Department of Psychology and Center for Human Growth and Development, University of Michigan, Ann Arbor; the National Institute of Mental Health, Bethesda, Md.; University of Southampton, Southampton, U.K.; and the Mount Sinai School of Medicine, New York. Address correspondence and reprint requests to Dr. Monk, Department of Psychology, University of Michigan, 2000 East Hall, 530 Church St., Ann Arbor, MI 48109; csmonk@umich.edu (e-mail).

Supported in part by NIMH grant K22 MH-068017 to Dr. Monk.

The authors thank Harvey Iwamoto for programming and computer support; Ken Towbin, M.D., and Alan Zametkin, M.D., for medical oversight; Eva Telzer, B.S., for data processing assistance; and Robert Cox, Ph.D., and Ziad Saad, Ph.D., for statistical and technical advice.

\section{References}

1. Pine DS, Cohen P, Gurley D, Brook J, Ma Y: The risk for earlyadulthood anxiety and depressive disorders in adolescents with anxiety and depressive disorders. Arch Gen Psychiatry 1998; 55:56-64

2. Thomas KM, Drevets WC, Dahl RE, Ryan ND, Birmaher B, Eccard $\mathrm{CH}$, Axelson D, Whalen PJ, Casey BJ: Amygdala response to fearful faces in anxious and depressed children. Arch Gen Psychiatry 2001; 58:1057-1063

3. Kringelbach ML, Rolls ET: Neural correlates of rapid reversal learning in a simple model of human social interaction. Neuroimage 2003; 20:1371-1383

4. Blair RJ, Cipolotti L: Impaired social response reversal. A case of 'acquired sociopathy'. Brain 2000; 123:1122-1141

5. Blair RJR, Morris JS, Frith CD, Perrett DI, Dolan RJ: Dissociable neural responses to facial expressions of sadness and anger. Brain 1999; 122:883-893

6. Blair RJ: The roles of orbital frontal cortex in the modulation of antisocial behavior. Brain Cogn 2004; 55:198-208

7. Bystritsky A, Pontillo D, Powers M, Sabb FW, Craske MG, Bookheimer SY: Functional MRI changes during panic anticipation and imagery exposure. Neuroreport 2001; 12:3953-3957

8. Fredrikson M, Fischer H, Wik G: Cerebral blood flow during anxiety provocation. J Clin Psychiatry 1997; 58(suppl 16):16-21

9. Hariri AR, Mattay VS, Tessitore A, Fera F, Weinberger DR: Neocortical modulation of the amygdala response to fearful stimuli. Biol Psychiatry 2003; 53:494-501

10. Monk CS, McClure EB, Nelson EE, Zarahn E, Bilder RM, Leibenluft E, Charney DS, Ernst M, Pine DS: Adolescent immaturity in attention-related brain engagement to emotional facial expressions. Neurolmage 2003; 20:420-428

11. Rauch SL, Savage CR, Alpert NM, Miguel EC, Baer L, Breiter HC, Fischman AJ, Manzo PA, Moretti C, Jenike MA: A positron emission tomographic study of simple phobic symptom provocation. Arch Gen Psychiatry 1995; 52:20-28

12. Rauch SL, Savage CR, Alpert NM, Fischman AJ, Jenike MA: The functional neuroanatomy of anxiety: a study of three disorders using positron emission tomography and symptom provocation. Biol Psychiatry 1997; 42:446-452

13. Tillfors M, Furmark T, Marteinsdottir I, Fischer H, Pissiota A, Langstrom B, Fredrikson M: Cerebral blood flow in subjects with social phobia during stressful speaking tasks: a PET study. Am J Psychiatry 2001; 158:1220-1226

14. Stein MB, Goldin PR, Sareen J, Zorrilla LT, Brown GG: Increased amygdala activation to angry and contemptuous faces in generalized social phobia. Arch Gen Psychiatry 2002; 59:10271034

15. Shin LM, Wright $\mathrm{Cl}$, Cannistraro PA, Wedig MM, McMullin K, Martis B, Macklin ML, Lasko NB, Cavanagh SR, Krangel TS, Orr SP, Pitman RK, Whalen PJ, Rauch SL: A functional magnetic resonance imaging study of amygdala and medial prefrontal cortex responses to overtly presented fearful faces in posttraumatic stress disorder. Arch Gen Psychiatry 2005; 62:273-281

16. Pine DS, Mogg K, Bradley BP, Montgomery LA, Monk CS, McClure EB, Guyer A, Ernst M, Charney DS, Kaufman J: Attention bias to threat in maltreated children: implications for vulnerability to stress-related psychopathology. Am J Psychiatry 2005; 162:291-296

17. Vasey MW, Daleiden EL, Williams LL, Brown LM: Biased attention in childhood anxiety disorders: a preliminary study. J Abnorm Child Psychol 1995; 23:267-279

18. Dalgleish T: Performance on the emotional Stroop task in groups of anxious, expert, and control subjects: a comparison of computer and card presentation formats. Cogn Emotion 1995; 1995:341-362

19. Taghavi MR, Neshat-Doost HT, Moradi AR, Yule W, Dalgleish T: Biases in visual attention in children and adolescents with clinical anxiety and mixed anxiety-depression. J Abnorm Child Psychol 1999; 27:215-223 
20. Mogg K, Bradley BP, Millar N, White J: A follow-up study of cognitive bias in generalized anxiety disorder. Behav Res Ther 1995; 33:927-935

21. Bradley BP, Mogg K, White J, Groom C, de Bono J: Attentional bias for emotional faces in generalized anxiety disorder. $\mathrm{Br}$ J Clin Psychol 1999; 38:267-278

22. Mogg K, Philippot P, Bradley BP: Selective attention to angry faces in clinical social phobia. J Abnorm Psychol 2004; 113: 160-165

23. Mogg K, Millar N, Bradley BP: Biases in eye movements to threatening facial expressions in generalized anxiety disorder and depressive disorder. J Abnorm Psychol 2000; 109:695-704

24. Birmaher B, Axelson DA, Monk K, Kalas C, Clark DB, Ehmann $\mathrm{M}$, Bridge J, Heo J, Brent DA: Fluoxetine for the treatment of childhood anxiety disorders. J Am Acad Child Adolesc Psychiatry 2003; 42:415-423

25. Kendall PC, Flannery-Schroeder E, Panichelli-Mindel SM, Southam-Gerow M, Henin A, Warman M: Therapy for youths with anxiety disorders: a second randomized clinical trial. J Consult Clin Psychol 1997; 65:366-380

26. Research Unit on Pediatric Psychopharmacology Anxiety Study Group: Fluvoxamine for the treatment of anxiety disorders in children and adolescents. N Engl J Med 2001; 344:1279-1285

27. The Pediatric Anxiety Rating Scale (PARS): development and psychometric properties. J Am Acad Child Adolesc Psychiatry 2002; 41:1061-1069

28. Mogg K, Bradley BP: Some methodological issues in assessing attentional biases for threatening faces in anxiety: a replication study using a modified version of the probe detection task. Behav Res Ther 1999; 37:595-604

29. Cox RW: AFNI: software for analysis and visualization of functional magnetic resonance neuroimages. Comput Biomed Res 1996; 29:162-173

30. Cohen MS: Parametric analysis of fMRI data using linear systems methods. Neuroimage 1997; 6:93-103
31. O'Doherty J, Dayan P, Schultz J, Deichmann R, Friston K, Dolan RJ: Dissociable roles of ventral and dorsal striatum in instrumental conditioning. Science 2004; 304:452-454

32. Rissman J, Eliassen JC, Blumstein SE: An event-related fMRI investigation of implicit semantic priming. J Cogn Neurosci 2003; 15:1160-1175

33. Rauch SL, van der Kolk BA, Fisler RE, Alpert NM, Orr SP, Savage CR, Fischman AJ, Jenike MA, Pitman RK: A symptom provocation study of posttraumatic stress disorder using positron emission tomography and script-driven imagery. Arch Gen Psychiatry 1996; 53:380-387

34. Vasa RA, Grados M, Slomine B, Herskovits EH, Thompson RE, Salorio C, Christensen J, Wursta C, Riddle MA, Gerring JP: Neuroimaging correlates of anxiety after pediatric traumatic brain injury. Biol Psychiatry 2004; 55:208-216

35. Davidson RJ: Well-being and affective style: neural substrates and biobehavioural correlates. Philos Trans R Soc Lond B Biol Sci 2004; 359:1395-1411

36. Milad MR, Quirk GJ: Neurons in medial prefrontal cortex signal memory for fear extinction. Nature 2002; 420:70-74

37. Neill DB: Frontal-striatal control of behavioral inhibition in the rat. Brain Res 1976; 105:89-103

38. Ferry AT, Ongur D, An X, Price JL: Prefrontal cortical projections to the striatum in macaque monkeys: evidence for an organization related to prefrontal networks. J Comp Neurol 2000; 425:447-470

39. Gigg J, Tan AM, Finch DM: Glutamatergic excitatory responses of anterior cingulate neurons to stimulation of the mediodorsal thalamus and their regulation by GABA: an in vivo iontophoretic study. Cereb Cortex 1992; 2:477-484

40. Hariri AR, Bookheimer SY, Mazziotta JC: Modulating emotional responses: effects of a neocortical network on the limbic system. Neuroreport 2000; 11:43-48

41. O'Doherty JP: Reward representations and reward-related learning in the human brain: insights from neuroimaging. Curr Opin Neurobiol 2004; 14:769-776 